\title{
Cooper Instability of Composite Fermions
}

\author{
Vito W. Scarola, Kwon Park, and J.K. Jain \\ Department of Physics, 104 Davey Laboratory, The Pennsylvania State University, University \\ Park, Pennsylvania 16802
}

(October 31, 2018)

When confined to two dimensions and exposed to a strong magnetic field, electrons screen the Coulomb interaction in a topological fashion; they capture and even number of quantum vortices and transform into particles called 'composite fermions' [1 3]. The fractional quantum Hall effect [4] occurs in such a system when the ratio (or 'filling factor', $\nu$ ) of the number of electrons and the degeneracy of their spin-split energy states (the Landau levels) takes on particular values. The Landau level filling $\nu=1 / 2$ corresponds to a metallic state in which the composite fermions form a gapless Fermi sea [5 [8]. But for $\nu=5 / 2$, a fractional quantum Hall effect is observed instead [9,10]; this unexpected result is the subject of considerable debate and controversy [11]. Here we investigate the difference between these states by considering the theoretical problem of two composite fermions on top of a fully polarized Fermi sea of composite fermions. We find that they undergo Cooper pairing to form a $p$-wave bound state at $\nu=5 / 2$, but not at $\nu=1 / 2$. In effect, the repulsive Coulomb interaction between electrons is overscreened in the $\nu=5 / 2$ state by the formation of composite fermions, resulting in a weak, attractive interaction.

The most important property of composite fermions is that they do not experience the external magnetic field $B$ but rather a drastically reduced magnetic field $B^{*}=B-2 p \rho \phi_{0}$. Here $\rho$ is the two-dimensional density of fermions, $2 p$ is the number of vortices, carried by the composite fermion, often intuitively envisioned as $2 p$ flux quanta, and $\phi_{0}=h / e$ is a flux quantum. In effect, each electron absorbs $2 p$ flux quanta of the external field to turn into a composite fermion. Electrons confined in two dimensions have unusual properties 
in a magnetic field; considerable progress towards understanding these properties has been made by modeling the composite fermion $(\mathrm{CF})$ system as an non-interacting gas of composite fermions [1:2]. In particular, the fractional quantum Hall effect (FQHE) [4] is a manifestation of the integral quantum Hall effect of composite fermions [3], and the metallic, non-FQHE state at the half-filled lowest Landau level is a Fermi sea of composite fermions [5 [ 8 ].

The Landau level filling $\nu=5 / 2=2+1 / 2$ corresponds to half-filled second LL. Here, both spin states of the lowest Landau level (LL) are completely occupied, contributing 2 to the filling factor. The fully occupied LL is treated as inert in this work, and the electrons in the partially filled second LL are assumed to be fully spin-polarized. These are valid approximations in sufficiently high magnetic fields. In complete analogy to the half-filled lowest LL, the model of non-interacting composite fermions would predict a Fermi sea of composite fermions at $\nu=5 / 2$ as well. However, experiments reveal a FQHE state here [9:10]. In fact, $5 / 2$ is the only even-denominator fraction to be observed in a single-layer system, and its physical origin has been a subject of debate and controversy [11].

To understand the fundamental difference between $\nu=5 / 2$ and $\nu=1 / 2$, it is necessary to go beyond the model of non-interacting composite fermions. Our theoretical investigations of the inter-CF interaction employ the Jain wavefunctions for composite fermions [1] [3]. These wavefunctions not only give an accurate quantitative account of the inter-CF interaction, but even capture the subtle, interaction-driven Wigner and Bloch instabilities of the $\mathrm{CF}$ liquid at small filling factors [12,13]; such instabilities are analogous to those believed to occur for the ordinary electrons gas (jellium) at low densities [14].

Central to this work is the following question, in analogous to the Cooper problem for ordinary superconductivity: if we begin by assuming a Fermi sea of composite fermions both at $\nu=1 / 2$ and $\nu=5 / 2$ and add two composite fermions at the Fermi surface, will they form a bound state? We find that the CF-Fermi sea is unstable to pairing of composite fermions at $\nu=5 / 2$ but not at $\nu=1 / 2$, as shown schematically in Fig. (四). We stress that, in contrast to the Bardeen-Cooper-Schrieffer (BCS) theory, we do not assume any attractive interaction, phonon-mediated or otherwise; the only interaction in the problem 
is the repulsive Coulomb interaction between electrons. However, the Coulomb interaction translates into a weak attractive interaction between composite fermions at $\nu=5 / 2$.

We work in the spherical geometry [15,16], in which $\mathrm{N}$ electrons are considered to move on the surface of a sphere under the presence of a radial magnetic field produced by a magnetic monopole of strength $Q$ at the centre. The flux through the surface of the sphere is $2 Q \phi_{0}$, where $2 Q$ is an integer, according to Dirac's quantization condition. The composite fermion theory maps the problem of interacting electrons at $Q$ to that of composite fermions at $Q^{*}=Q-N+1$ (assuming here and below composite fermions of vorticity $2 p=2$ ). The Jain wavefunctions for interacting electrons at $Q$ are given by $\Psi_{Q}=\mathcal{P}_{L L L} \Phi_{1}^{2} \Phi_{Q^{*}}$, where $\Phi_{Q^{*}}$ are wavefunctions of non-interacting electrons at $Q^{*}$, and $\Phi_{1}$ is the wavefunction of the fully occupied lowest Landau level at $Q_{1}=(N-1) / 2$, and $\mathcal{P}_{L L L}$ is the lowest LL projection operator. Due to the rotational symmetry, the total orbital angular momentum $L$ is a good quantum number, preserved in going from $\Phi_{Q^{*}}$ to $\Psi_{Q}$.

We are interested in composite fermions in a vanishing effective magnetic field, that is, when $Q^{*}=0$, which is obtained at $Q=N-1$. Here, for $N=n^{2}$, the ground state $\Psi_{Q}\left(\Phi_{Q^{*}}\right)$ has uniform density $(L=0)$, as it contains $n$ filled shells of composite fermions (electrons). We approach the CF Fermi sea as the $N \rightarrow \infty$ limit of the filled shell states. The systems with a $\mathrm{CF}$ pair occur at $Q^{*}=0$ for $N=n^{2}+2$, corresponding to situation when two $\mathrm{CF}$ particles are added to the $(n+1)$ st shell. The individual angular momenta of the additional particles are $l=n$, implying that there is one multiplet at each total angular momentum $L=1,3, \ldots, L_{\max }=2 n-1$ with a degeneracy of $2 L+1$. The wavefunction of a CF pair at $B^{*}=0$ for a given $L$ is $\Psi_{L}^{C F-p a i r}=\mathcal{P}_{L L L} \Phi_{1}^{2} \Phi_{L}^{e l-p a i r}$, where $\Phi_{L}^{\text {el-pair }}$ is the wavefunction of an electron pair at $B=0$. We will also consider a pair of $\mathrm{CF}$, corresponding to two holes in the $n$th shell at $Q^{*}=0 . \Psi_{L}^{C F-\text { pair }}$ contains no adjustable parameter.

For $\Phi_{L}^{e l-p a i r}$, the Coulomb energy of the electron pair, which is proportional to the average inverse distance between the two electrons in the otherwise empty shell, decreases with $L$, in accordance with Hund's rule of atomic physics [17]. This implies that the the smallest $L$ ( $L=1$ for fully polarized particles) corresponds to the smallest distance between two 
electrons of a pair. By analogy, the size of the CF pair in $\Psi_{L}^{C F-\text { pair }}$ also increases with $L$. We have confirmed this by monitoring the influence of an additional short-range interaction to the pair energy.

As it stands, $\Psi_{L}^{C F-\text { pair }}$ is written in the lowest Landau level, that is, for $\nu=1 / 2$. In order to treat $\nu=5 / 2$, we use the method of Park et al. 18 to map the problem of the Coulomb interaction in the second $(s=1)$ Landau level into that of an effective interaction $V^{e f f}(r)$ in the lowest $(s=0)$ Landau level. $V^{e f f}(r)$ is chosen so that the two interactions have the same Haldane pseudopotentials [15] that is, $V_{1, m}=V_{0, m}^{e f f}$. The parameters $V_{s, m}$ are the interaction energies of two particles in sth LL in relative angular momentum $m$ state (in the planar geometry) and completely specify the interparticle interaction in the sth Landau level.

The energy of $\Psi_{L}^{C F-p a i r}, E[L]$, is evaluated in a Monte Carlo approach [19]. Figure (2) shows the energy of the pair as a function of $L$ for electrons at $B=0$, and for composite fermions at $\nu=1 / 2$ and $5 / 2$. The interaction energy between the two added electrons decreases with increasing $L$ at $B=0$, as expected. Similar behavior is found for composite fermions at $\nu=1 / 2$. However, the opposite behavior is seen at $\nu=5 / 2$, indicating an attractive interaction between composite fermions at $\nu=5 / 2$. The largest binding energy is obtained for the $L=1$ channel. This qualitative difference between the physics at $\nu=1 / 2$ and $\nu=5 / 2$ is the principal result of this work.

The estimation of the thermodynamic limit of the binding energy from our finite size study can be difficult. When the pair interaction energy is not small compared to the intershell spacing, many shells would participate in the pair wavefunction, and our approximation of restricting the pair to the lowest unoccupied shell would break down. A determination of the appropriate 'parent' wavefunction $\Phi^{e l-p a i r}$ is obviously quite complicated in this regime, and consequently, so is obtaining $\Psi^{C F-\text { pair }}$. However, past studies 20 have shown that distinct excitations in $\Phi$ with the same quantum numbers may produce the same excitation $\Psi$, because the Hilbert space at $\nu=1 / 2$ is greatly restricted compared to that at zero 
magnetic field. Therefore, as a first step, we proceed without any explicit consideration of shell mixing. We have studied up to $n=6$ filled shells; both CF-particle pairs and CF-hole pairs are considered for up to $n=5$ and only the latter for $n=6$. Figure (3) shows that despite a substantial decrease with $N$, the binding energy $\Delta[L=1]=E[L=1]-E\left[L_{\max }\right]$ at $\nu=5 / 2$ still extrapolates to a non-zero negative value of $-0.0035( \pm 0.0013) e^{2} / \epsilon l_{0}$ as $N \rightarrow \infty$, where $l_{0}=\sqrt{\hbar / e B}$ and $\epsilon$ is the dielectric constant of the background material. After taking account of the transverse width of the electron wavefunction 21,22, the binding energy in the $L=1$ channel is estimated to be $-0.0025( \pm 0.0015) e^{2} / \epsilon l_{0}$ for densities in the range $(0.5-3.0) \times 10^{11} \mathrm{~cm}^{-2}$. Following the weak-coupling BCS theory, it is natural to identify $2|\Delta|$ with the energy gap of the FQHE state at $\nu=5 / 2$. For the parameters of the experiment of Pan et al. [10], our calculated $2|\Delta|$ for $L=1$ is $0.5( \pm 0.3) \mathrm{K}$, which turns out to be on the same order of magnitude as the measured gap of about $0.1 \mathrm{~K}$. While this is encouraging, we note that the effect of shell mixing is an important issue, and must be considered to ascertain the quantitative reliability of our preliminary estimate.

The appearance of pairing may seem surprising in a model with strong repulsive interaction. However, the Coulomb repulsion is overcome through the formation of composite fermions, which screens out the Coulomb interaction quite effectively, to the extent that a total neglect of the interaction between composite fermions is sufficient for many purposes. Furthermore, the screening takes place in a topologically rigid manner, independent of the interaction strength or the Landau level index, through the binding of precisely two vortices to each electron. Therefore, it is plausible that sometimes an overscreening of the Coulomb interaction occurs, producing an effectively attractive interaction between composite fermions. The reason that there is an attraction at $\nu=5 / 2$ but not at $\nu=1 / 2$ is that the matrix elements for the Coulomb interaction in the second Landau level are weaker than in the lowest LL because of the greater spread of the electron wavefunction in the former, especially at short distances. For example, $V_{1,1} / V_{0,1}=0.93$. This slight softening of the inter-electron repulsive interaction in the second LL is sufficient to make the inter-CF interaction weakly negative. 
There has been earlier work on pairing of composite fermions. Many years ago, Greiter, Wen, and Wilczek [23] argued for $p$-wave pairing of composite fermions at $\nu=1 / 2$ and $5 / 2$ within a Chern-Simons formulation of composite fermions. The Chern-Simons method, however, is quantitatively unreliable in this application because of its inadequacy in describing the energetics or the short-distance behavior. Even within this approach, Bonesteel [24] has noted that a pair breaking term not considered in ref. 23] may potentially alter its conclusion. Greiter et al. further suggested that the paired CF state may be described in terms of a Pfaffian wavefunction written by Moore and Read [25]. Recent exact diagonalization [26,27] and variational [18] studies have provided support for the validity of a Pfaffian-like wavefunction at $\nu=5 / 2$.

The pairing of composite fermions at $\nu=5 / 2$ has a topological origin, and occurs in spite of strong repulsive interaction between electrons. The repulsion is circumvented because the objects that pair up are not electrons but composite fermions. We speculate that a fundamental reorganization of the state, for example, creation of new quasiparticles, must happen in any system in order for pairing to ensue, starting from repulsive interactions alone. This is indeed the case in several theoretical models of high-temperature superconductivity, where the pairing is believed also to be caused by repulsive interactions. 


\section{REFERENCES}

[1] Heinonen, O. (ed.) Composite Fermions (World Scientific, New York, 1998).

[2] Das Sarma, S. \& Pinczuk, A. (eds) Perspectives in Quantum Hall Effects (Wiley, New York, 1997).

[3] Jain, J.K. Composite-fermion approach for the fractional quantum Hall effect. Phys. Rev. Lett. 63, 199-202 (1989).

[4] Tsui, D.C., Stormer, H.L. and Gossard, A.C. Two-Dimensional Magnetotransport in the Extreme Quantum Limit. Phys Rev. Lett. 48, 1559-1562 (1982)

[5] Halperin, B.I., Lee, P.A. and Read, N. Theory of the half-filled Landau level. Phys. Rev. B 47, 7312-7343 (1993).

[6] Willett, R.L. et al. Experimental Demonstration of a Fermi Suface at One-Half Filling of the Lowest Landau Level. Phys Rev. Lett. 71, 3846-3849 (1993).

[7] Goldman, V.J. et al. Detection of Composite Fermions by Magnetic Focusing. Phys Rev. Lett. 72, 2065-2068 (1994).

[8] Kang, W. et al. How Real Are Composite Fermions? Phys. Rev. Lett. 71, 3850-3853 (1993).

[9] Willett, R.L. et al. Observation of an even-denominator quantum number in the fractional quantum hall effect. Phys. Rev. Lett. 59, 1779-1782 (1987).

[10] Pan, W. et al. Exact Quantization of the Even-Denominator Fractional Quantum Hall State at $\nu=5 / 2$ Landau Level Filling Factor. Phys. Rev. Lett. 83, 3530-3533 (1999).

[11] Eisenstein, J.P. in Perspectives in Quantum Hall Effects (eds Das Sarma, S. \& Pinczuk A.) 37-70 (Wiley, New York, 1997).

[12] Kamilla, R.K. and Jain, J.K. Excitonic instability and termination of fractional quantum Hall effect. Phys. Rev. B 55, R13417-R13420 (1997). 
[13] Park, K. and Jain, J.K. Spontaneous magnetization of composite fermions. Phys. Rev. Lett. 83, 5543-5546 (1999).

[14] Tanatar, B. and Ceperley, D.M. Ground state of the two-dimensional electron gas. Phys. Rev. B 39, 5005-5016 (1989).

[15] Haldane, F.D.M. Fractional Quantization of the Hall Effect: A Hierarchy of Incompressible Quantum Fluid States. Phys. Rev. Lett. 51, 605-608 (1983).

[16] Wu, T.T. and Yang, C.N. Dirac monopole without string: Monopole harmonics. Nucl. Phys. B 107, 365-380 (1976).

[17] Wojs, A. and Quinn, J.J. Hund's rule for monopole harmonics, or why the composite fermion picture works. Solid State Comm. 110, 45-49 (1999).

[18] Park, K., Melik-Alaverdian, V., Bonesteel, N.E. and Jain, J.K. Possibility of $p$-wave pairing of composite fermions at $\nu=1 / 2$. Phys. Rev. B 58, R10167-R10170 (1998).

[19] Jain, J.K. and Kamilla, R.K. Composite fermions in the Hilbert space of the lowest electronic Landau level. Int. J. Mod. Phys. B 11, 2621-2660 (1997).

[20] Wu, X.G., Jain, J.K. Excitation spectrum and collective modes of composite fermions. Phys. Rev. B 51, 1752-1761 (1995).

[21] Fang, F.F. and Howard, W.E. Negative field-effect mobility on (100) Si surfaces. Phys. Rev. Lett. 16, 797-800 (1966);

[22] Zhang, F.C. and Das Sarma, S. Excitation gap in the fractional quantum Hall effect: Finite layer thickness corrections. Phys. Rev. B. 33, 2903-2906 (1986).

[23] Greiter, M., Wen, X.G. and Wilczek, F. Paired Hall states. Nucl. Phys. B 374 567-614 (1992).

[24] Bonesteel, N.E. Singular Pair Breaking in the Composite Fermi Liquid Description of the Half-Filled Landau Level. Phys. Rev. Lett. 82, 984-987 (1999). 
[25] Moore, G. and Read, N. Nonabelions in the fractional quantum Hall effect Nucl. Phys. B 360, 362-396 (1991).

[26] Morf, R. Transition from Quantum Hall to Compressible States in the Second Landau Level: New Light on the $\nu=5 / 2$ Enigma. Phys. Rev. Lett. 80, 1505-1508 (1998).

[27] Rezayi, E.H. and Haldane, F.D.M. Transition from Paired Quantum Hall to Compressible States at the Half Filling of the Lowest Two Landau Levels. Preprint condmat/9906137 at ihttp://xxx.lanl.gov; (1999).

\section{Acknowledgements}

This work was supported in part by the National Science Foundation. We thank the Numerically Intensive Computing Group led by V. Agarwala, J. Holmes, and J. Nucciarone, at the Penn State University CAC, for assistance and computing time with the LION-X cluster. 


\section{FIGURES}
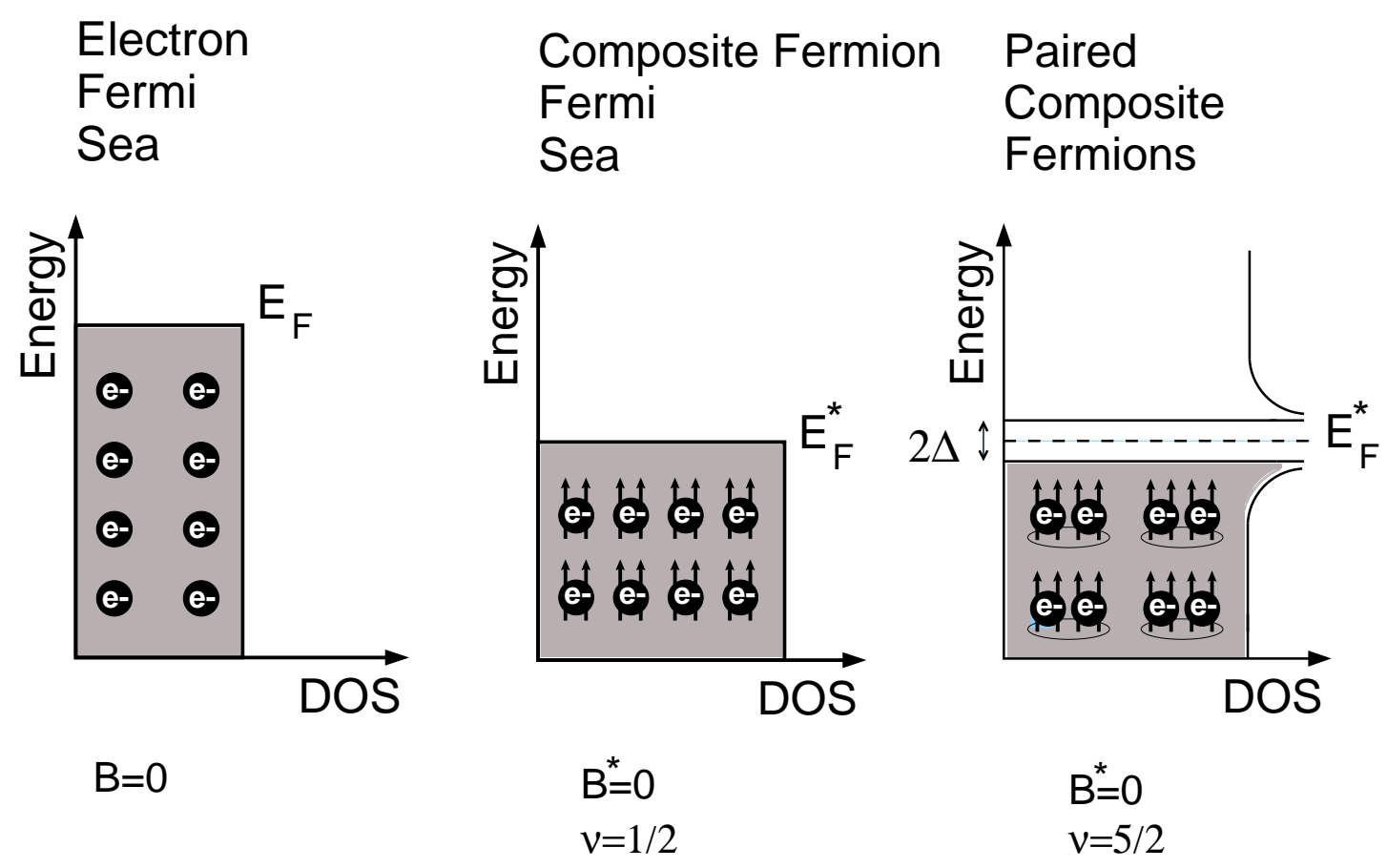

FIG. 1. Density of States (DOS) for electrons and composite fermions at zero effective magnetic flux. Left panel, electrons at $B=0$. Centre panel, composite fermions at $\nu=1 / 2$. Right panel, pairing of composite fermions at $\nu=5 / 2$. The composite fermions are shown as electrons carrying two flux quanta. 


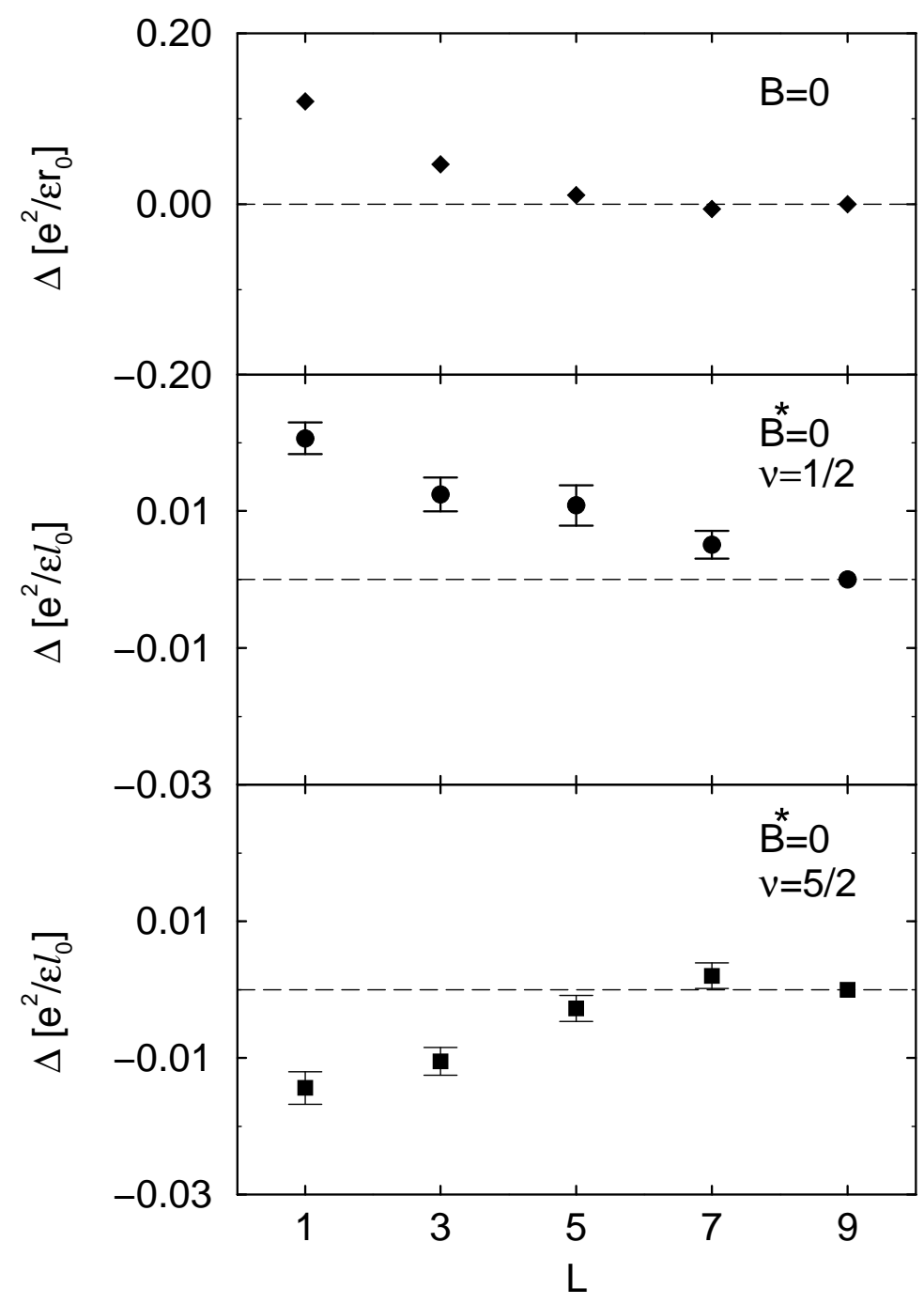

FIG. 2. The interaction energy of pairs of electrons and pairs of composite fermions at zero effective magnetic flux as a function of angular momentum $L$. The results are shown for a system with $N=27$ particles. Top panel, electrons; middle panel composite fermions at $\nu=1 / 2$; bottom panel, composite fermions of $\nu=5 / 2$. The quantity $l_{0}=\sqrt{\hbar / e B}$ is the magnetic length, $r_{0}=(\pi \rho)^{-1 / 2}$ is the average interparticle separation, and $\epsilon$ is the dielectric constant of the background material $(\epsilon=12.8$ for $\mathrm{GaAs})$. 


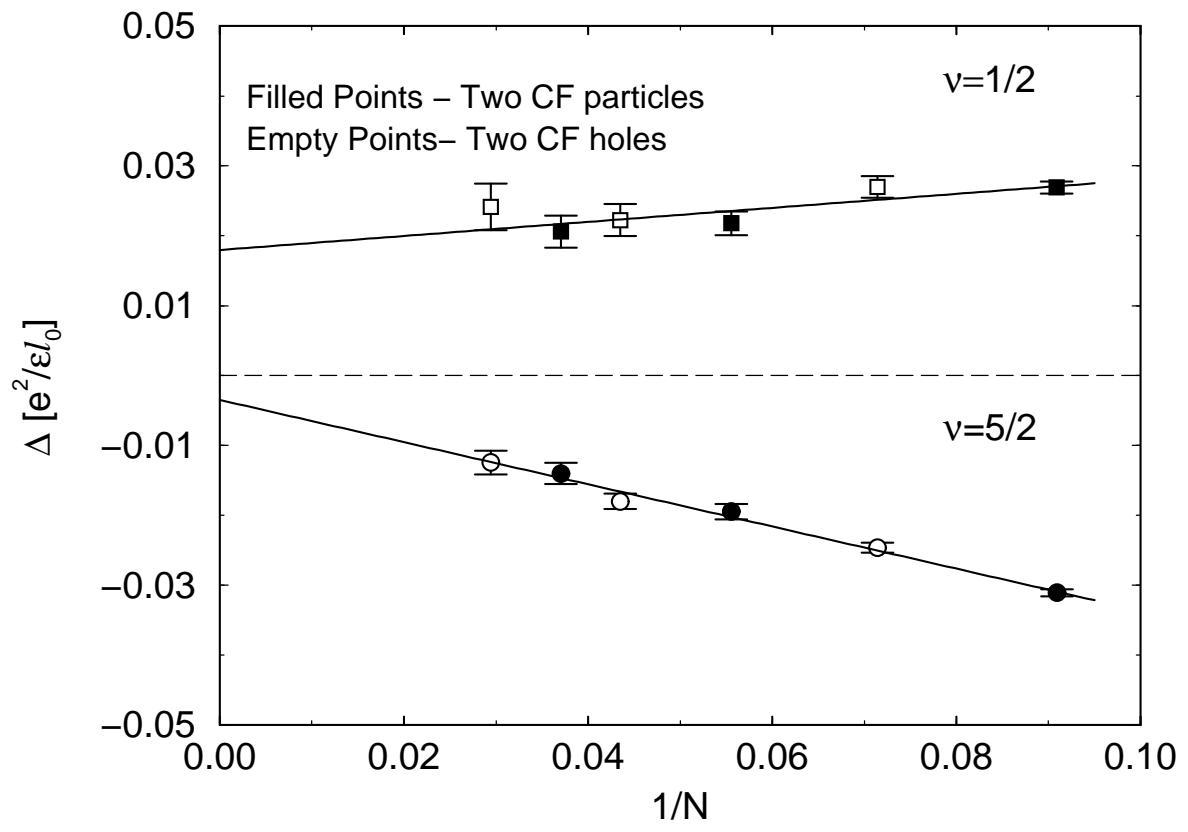

FIG. 3. The binding energy of the composite fermion (CF) pair in the $L=1$ channel at $\nu=1 / 2$ and $5 / 2$ as a function of $1 / N$. The binding energy is defined as the energy of the pair at $L=1$ relative to its energy at $L_{\max }$. The filled (empty) symbols are for CF-particles (CF-holes) on top of the CF-Fermi surface. As expected from particle-hole symmetry, satisfied by the wavefunctions considered here to an excellent approximation, the binding energies for the CF-particles and CF-holes fall on the same line. 